\title{
Synthesis of Nanopowders and Consolidation of Nanoceramics of Various Applications
}

\author{
Edwin Gevorkyan ${ }^{1}$, Dmitry Sofronov ${ }^{2}$, Sergiy Lavrynenko ${ }^{3 *}$ and Miroslaw Rucki ${ }^{4}$ \\ ${ }^{1}$ Ukrainian State University of Railway Transport, Kharkov, Ukraine \\ ${ }^{2}$ State Scientific Institution "Institute for Single Crystals" National Academy of Sciences of Ukraine \\ ${ }^{3}$ National Technical University "Kharkov Polytechnic Institute", Ukraine \\ ${ }^{4}$ Kazimierz Pulaski University of Technology and Humanities in Radom, Poland \\ Email: s_lavr@yahoo.com
}

\begin{abstract}
In the paper, some problems of nanoceramics production are discussed. Some crucial stages in the production cycle are pointed out, among others the nanoscale powder synthesis and the compaction during the sintering. A novel method of hot pressing under the directly applied electric current was proposed. Some examples of advantageous characteristics obtained through the new method were provided. The hot press sintering under the electric current is suitable for the production of structured nanoceramics, cutting tools for industrial applications, filters for industry and the functional nanoceramics like optical elements or biomedical implants.
\end{abstract}

Keywords: Nanoceramics, nanopowder, sintering, compaction.

\section{$1 \quad$ Introduction}

The nanostructured materials are definitely not new in the ceramic field, since the nature of the minerals employed in formulating the porcelain has resulted in 'nanoscale' features (the dimensions below $100 \mathrm{~nm}$ ) [1]. The development of physical synthesis by gas condensation initiated relatively recent research in the field of purposely nanostructured ceramics [2]. In the last decades, nanostructured materials were found suitable in many advanced technologies such as thermomechanics, aerospace, nuclear power plant and biomedicine [3], especially in form of nanoceramic and nanocomposite coatings [4] or thin films [5].

Preparation of nanomaterials like nanoceramics is a complex process starting from the synthesis of oxide nanoparticles precursors and ending with the sintering [6]. The process involves several major steps: a powder mix production with a bunch of powder and plasticizers for molding material, the formation of the workpiece, drying and calcining the preform, treating the obtained product (mechanical, thermal treatment and metallization). In some applications, the pulsed electrical current was found useful in two-step sintering process of transparent $\mathrm{Al}_{2} \mathrm{O}_{3}$ ceramics [7]. The properties of the resulting material to a large extent depend on the characteristics of the nanopowders used, foremost among which are the particle size, polydispersity and purity (contaminations content) [8].

One of the priorities for creating of new nanomaterials with specific functional properties is to search for a fundamentally new and improvement of existing technological solutions in the field of chemical synthesis of nanopowders and their subsequent consolidation in the solid substance. The paper is focused on the effective synthesis and consolidation technologies that may be applied for the sintering of the nanoceramics of various functional properties.

\section{Synthesis of Nanopowders}

Among a wide range of industrial and biomedical applications [9], nanoceramics developed by the authors of this project are suitable for the following ones:

1. Structural nanoceramics: 
a) Blades and elements of gas turbine and rotary engines, internal combustion engines, wind turbines;

b) Instrument.

2. Cutting tools (inserts and complex-tools);

3. Functional nanoceramics:

a) Optical elements of laser technology and infrared optics;

b) Sorbents for the purification of liquid media from radionuclides and heavy metals ions and salts;

c) Bioengineering ceramics for implants;

4. Filters for industry (e.g. for liquid aluminum cleaning) and others.

One of the most promising directions in the ceramic industry to provide a refractory ceramic material with high ductility based on titanium and zirconium dioxides. In addition, considerable practical interest is optical ceramic material which works in infrared spectrum based on the ZnS. This requires as starting material powders consisting of uniform monodisperse particles (the particle size ca. 10-200 nm). Therefore, the development of technology for synthesizing such powders is one of the major challenges to be addressed in the creation of ceramic materials with improved functional properties. Dimensional analysis, morphological and structural characteristics of the nanoparticles show that they are highly dependent on the method and conditions for obtaining nanostructures.

Recently, much attention of researchers in the field of synthesis of the compounds and the development of new materials is focused on the study of reactions in the microwave activation. This technology can increase the rate of chemical processes in several times, and also significantly increase the yield and purity of the nanopowder. A distinctive feature of microwave heating is to direct the energy absorption material that serves to focus energy into a reaction zone and to achieve high temperature gradients, which provide a significant increase in the rates of chemical processes. Precipitation from aqueous solutions is simple and easily implemented in practice method of producing chalcogenides and metal oxides. The sizes of the finally obtained particles tend to be dependent on the $\mathrm{pH}$, concentration of components, the activation conditions, temperature etc.

Complex studies of the effect of preparation conditions of zinc sulfide, titanium dioxide and zirconium particles from aqueous solutions ( $\mathrm{pH}$, concentration of components, microwave activation, the nature of the starting metal salt of the anion etc.) on morphological and structural properties were conducted. Depending on conditions of synthesis and activation it appeared possible to obtain particles of different nanoscale sizes.

In order to prepare the solution $\mathrm{Zn}\left(\mathrm{NO}_{3}\right)_{2} \cdot 6 \mathrm{H}_{2} \mathrm{O}$, thiourea $\mathrm{SC}\left(\mathrm{NH}_{2}\right)_{2}$ was used and the $25 \%$ water solution of the ammonia $\mathrm{NH}_{3}$. The basis for the solutions was a distillated water. The precipitation of zinc sulfide was carried out from $0.1 \mathrm{M}$ solution of zinc nitrate. To $100 \mathrm{~mL}$ of the metal salt some ammonia solution was added until the required $\mathrm{pH}$ value was reached. The $\mathrm{pH}$ value was varied in the range from 8 up to 12. After that, thiourea was added in the molar proportion $c\left(\mathrm{SC}\left(\mathrm{NH}_{2}\right)_{2}\right): C\left(\mathrm{Me}^{2+}\right)=$ 1:1. The obtained reagent solution was heated until boiling, and then boiled for 1 hour. After the synthesis, the obtained deposit was filtered, washed with the distillated water several times and dried at the room temperature during 24 hours.

The synthesis with microwave activation was performed using the microwave device MARS type made by GEM Corporation Matthews, USA. $50 \mathrm{~mL}$ of the $0.1 \mathrm{M}$ zinc nitrate solution of the required $\mathrm{pH}$ value was placed in the Vial for the microwave synthesis, and the thiourea was added in the molar proportion $\mathrm{Me}^{2+} / \mathrm{SC}\left(\mathrm{NH}_{2}\right)_{2}=1: 1$. The treatment in the microwave field lasted for 30 minutes, and then the deposit was filtered, washed and dried.

The titanium dioxide was obtained through the decomposition of titanium hydroxide precursor at the temperature $650^{\circ} \mathrm{C}$. When titanium was solved, to the colored solution some nitric acid was added drop by drop until the color completely disappeared. Next, the $25 \%$ ammonia solution was added, portions of $5 \mathrm{~mL}$, until the pH 8-12 was obtained. Then the deposit was filtered, dried, and afterwards was heated at $650^{\circ} \mathrm{C}$ during 4 hours. This way the white powder of titanium dioxide was produced.

Morphology of the obtained powders surfaces was analyzed using the scanning microscope (SEM) JSM-6390LV. As examples in the Figures 1-3 present the zinc sulfide particles micrographs formed with different $\mathrm{pH}$, concentration of the sulfonating reagent (thiourea), and activation method. 

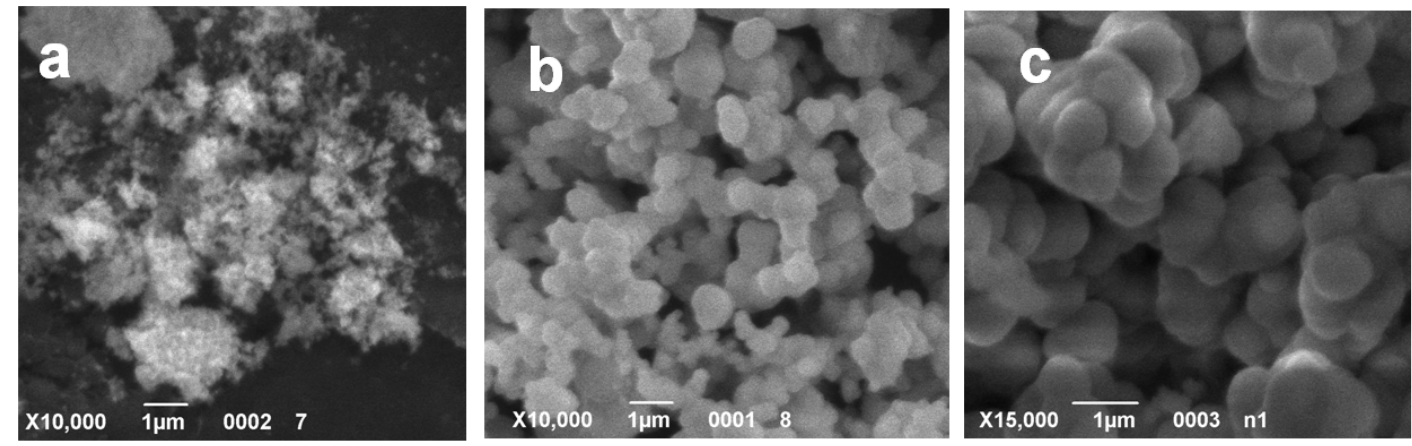

Figure 1. Photomicrographs of $\mathrm{ZnS}$ particles obtained by the ratio $\left.c\left(\mathrm{Zn}^{2+}\right): \mathrm{C}\left(\mathrm{SC}_{(\mathrm{NH}}\right)_{2}\right)$ 1:1 with a) p $\mathrm{H}$ 8; b) pH 10; and c) pH 12.
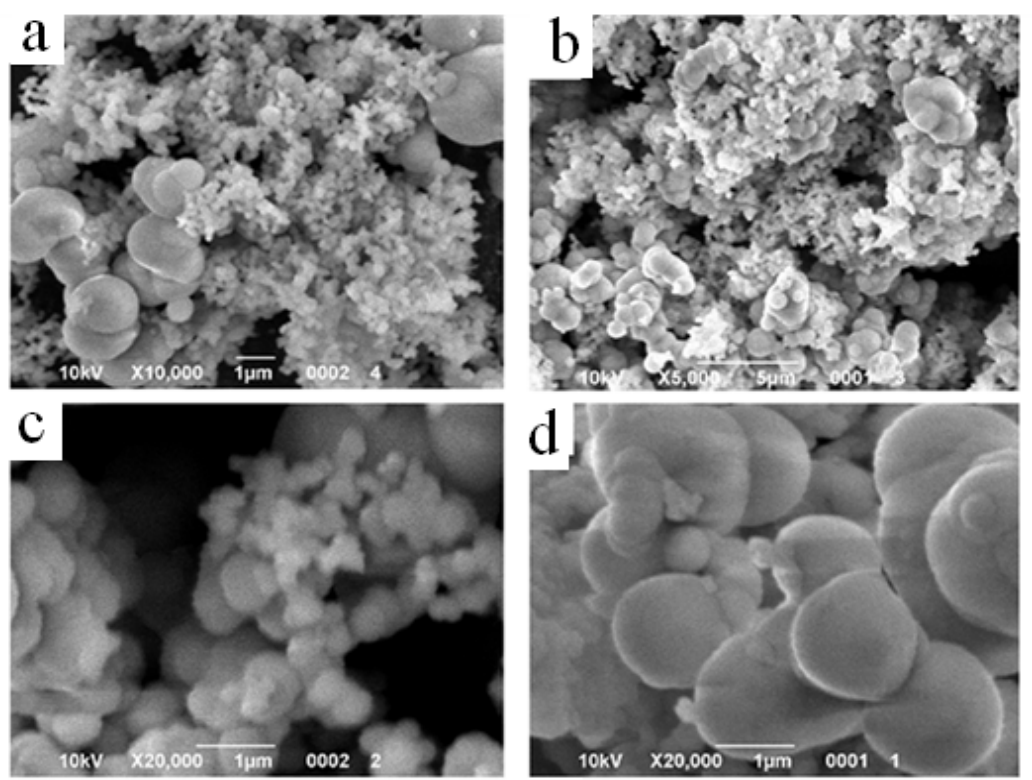

Figure 2. Photomicrographs of $\mathrm{ZnS}$ particles obtained by the ratio $C\left(\mathrm{SC}\left(\mathrm{NH}_{2}\right)_{2}\right): C\left(\mathrm{Zn}^{2+}\right) 1: 1$ with temperature $150^{\circ} \mathrm{C}$ (microwave activation): a) $\mathrm{pH} 8$; b) $\mathrm{pH} 9.5$; c) $\mathrm{pH} 10.5$; and d) $\mathrm{pH} 12$.
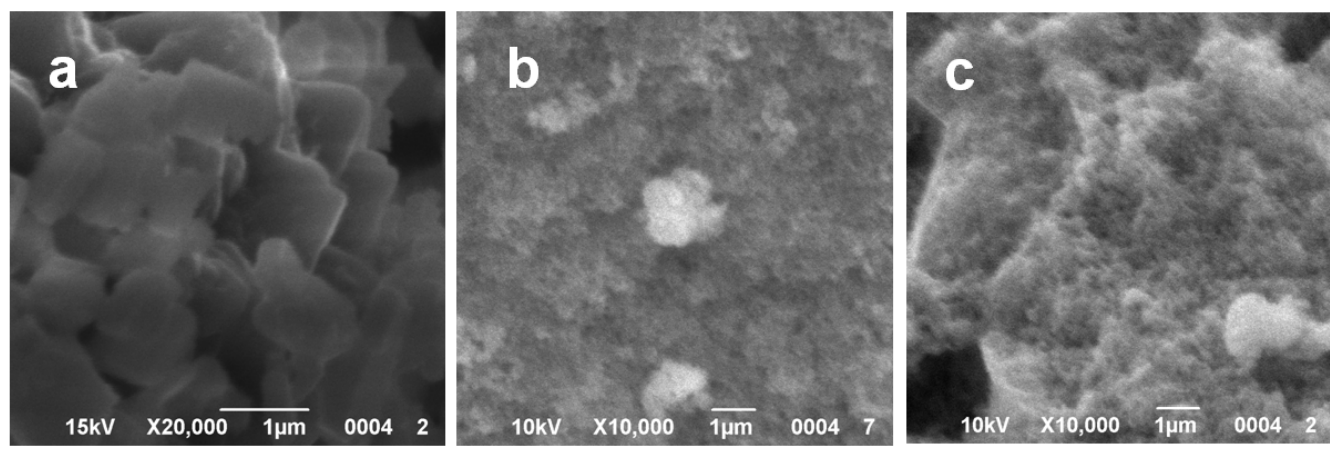

Figure 3. Photomicrographs of titanium dioxide particles obtained with a) pH 7.5; b) pH 9.1; and c) pH 10.3. 
The IR spectrum was obtained in the tablets of KBr with the Fourier IR-spectrometer SPECTRUM ONE (PerkinElmer). The X-ray analysis of the powders was carried out with the diffractometer Siemens D500 in the copper radiation with graphite monochromator at the secondary beam. The diagrams were measured in the angle range of $10<2 \theta<110^{\circ}$ with the step $0.02^{\circ}$ and collection time $10 \mathrm{~s}$ in each point. Figures 4 and 5 present the X-ray diagrams and IR spectra obtained for ZnS powders produced at different $\mathrm{pH}$ values.

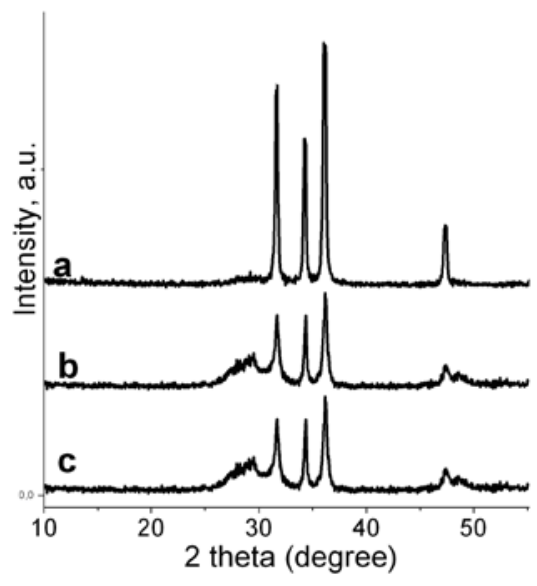

Figure 4. X-ray diagrams of ZnS particles obtained at different $\mathrm{pH}$ : a) $\mathrm{pH} 12$; b) pH 10; and c) pH 8 .

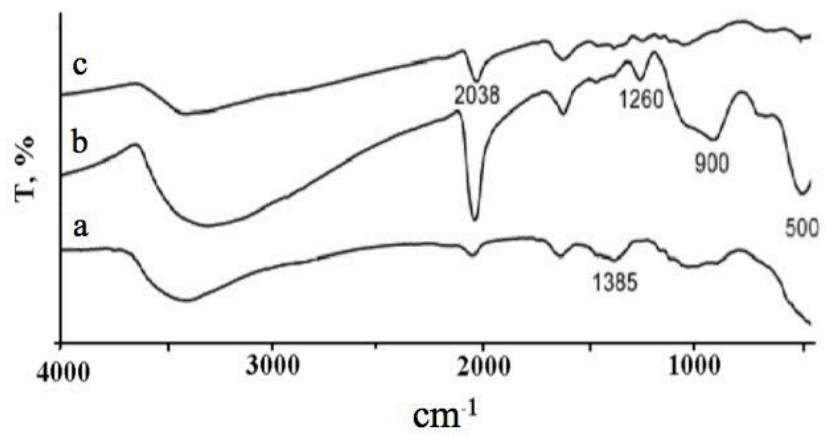

Figure 5. IR spectra of $\mathrm{ZnS}$ particles obtained at different pH: a) $\mathrm{pH}$ 8; b) pH 10; and c) pH 12 .

IR spectrum of the ZnS powder obtained at pH 8 (Fig. 5a) reveals absorption in the area of 3000-3600 $\mathrm{cm}^{-1}$ with its maximum in $3400 \mathrm{~cm}^{-1}$, as well as the absorption band at $1620 \mathrm{~cm}^{-1}$. They are bound with the valence and deformation fluctuations of the water molecules adsorbed on the ZnS particles surface, respectively. The absorption band $2038 \mathrm{~cm}^{-1}$ is related to the fluctuations of SCN. The absorption band $1385 \mathrm{~cm}^{-1}$ may be ascribed to the fluctuations of the ion $\mathrm{NO}_{3}^{-}$, and absorption band $900 \mathrm{~cm}^{-1}$ to the fluctuations of the bind $\mathrm{Zn}-\mathrm{OH}$.

The absorption band $1028 \mathrm{~cm}^{-1}$ is related to the symmetrical fluctuations of the thiourea's C-S. When $\mathrm{pH}$ is increased to 10.9 value (Fig. 5b), the increase of the bands 2038 and $900 \mathrm{~cm}^{-1}$ is observed, as well as the absorption bands of 1260,685 and $500 \mathrm{~cm}^{-1}$. The absorption band of $685 \mathrm{~cm}^{-1}$ is probably related to the fluctuations of S-O, and of $500 \mathrm{~cm}^{-1}$ with $\mathrm{Zn}-\mathrm{O}$ fluctuations. The absorption band of $1260 \mathrm{~cm}^{-1}$ is the consequence of thiourea adsorption on the surface of the ZnS particles and represents the fluctuations of N-SC-N. When $\mathrm{pH}$ is increased up to 12, practically all absorption bands become weakened (Fig. 5c), which indicates the decrease of the additions in the obtained $\mathrm{ZnS}$. The yield of zinc sulfite slightly increases with the increase of $\mathrm{pH}$ from 40 (at $\mathrm{pH} 8$ ) up to 45 (at $\mathrm{pH} 12$ ). 
The X-ray diagrams of the ZnS powders obtained with microwave activation at any $\mathrm{pH}$, are identical to the diagram of the $\mathrm{ZnS}$ powder obtained without it at $\mathrm{pH} 12$. On the other hand, the IR spectra are quite similar to the ones obtained for the powders produced without microwave activation. The most important difference is the lack of absorption band of $500 \mathrm{~cm}^{-1}$, which indicates the absence of the zinc oxide in the powder. The yield of zinc sulfite produced with the microwave activation is much higher, and reaches ca. $90 \%$ irrespectively of $\mathrm{pH}$ during the synthesis process.

Nanopowders $\mathrm{TiO}_{2}$ are usually prepared by sol-gel method, with the expensive titanium alkoxides $\left(\mathrm{Ti}\left(\mathrm{OC}_{2} \mathrm{H}_{5}\right)_{4}\right.$ or $\left.\mathrm{Ti}\left(\mathrm{OC}_{4} \mathrm{H}_{9}\right)_{4}\right)$ as the starting components. The authors suggested instead, the method of nanosized titanium dioxide fabrication by the decomposition of the precursor of titanium hydroxide obtained by aqueous deposition. The $\mathrm{TiO}_{2}$ particle sizes from 50 to $100 \mathrm{~nm}$ could be obtained, dependent on the conditions of the titanium hydroxide production.

\section{$3 \quad$ Electrical Consolidation}

The key task of the consolidation of nanomaterials is to achieve a high density of the material while maintaining nanoscale grains in the range keeping a nanosize effect. In the experience of the authors, the choice of the consolidation process depends on the structure of grain boundaries or interphase boundaries. The main difficulty in the technology of compact products out of the nanopowders, is the residual porosity [10]. Residual porosity and defect borders significantly affect the properties of nanostructured materials. The new methods of strain and composition nanostructuring make it possible to create new structural and functional materials for engineering, medicine and energetics.

The novel studies performed by the authors of this project covered the spherical-elemental multimodal model. The densely packed elementary composition of objects at the combined parameters of direct current and the external pressure has been developed, and the powder metallurgy phenomenology of electric effects in nanomaterials considered. Empirical evidence-based energy-saving background for electrical agglomeration of nanoceramics to bimodal, including refractory functional basis were also worked out.

Figure 6 presents the scheme of the device designed for the nanopowders consolidation through the hot pressing under the high voltage. In the investigations, the prefabricated samples of 20 mm diameter and $4 \mathrm{~g}$ weight were initially made out of the powders. The moulds were made out of the special graphite МПГ-7 type (according to the Russian nomenclature) that ensure the necessary strength at the pressure of 45-50 MPa. The temperature of consolidation was measured at this pressure, and the time of consolidation was 2 minutes. The alternating electric current of 1500-2000 A and 5-10 V was applied to support the consolidation process.

a)

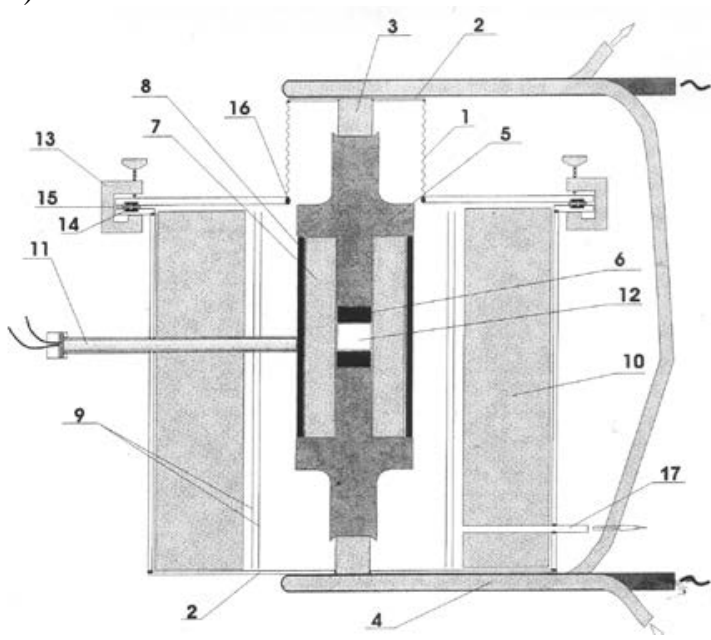

b)

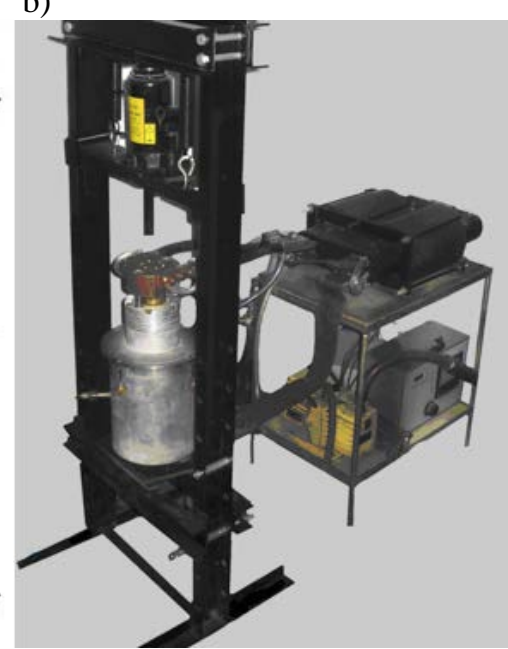

Figure 6. Hot-pressure electrical sintering device. 
Explanations to the Fig. 6: 1 - bellows X18H10, 2 - flanges X18H10T, 3 - copper conductors, 4 water-cooled conductors, 5 - die punch (graphite МПГ-7), 6 - graphite sealing, 7 - mould (graphite МПГ -7), 8 - sleeve, 9 - molybdenum screens, 10 - thermal isolation, 11 - thermocouple BP - 5/20, 12 - sintered sample, 13 - dielectrical cleats, 14 - sleeve, 15 - special dielectrical sleeve, 16 - vacuum welding of the contacts, 17 - vacuum outlet.

The object of the research was a refractory ceramic, genesis and destruction of nanostructures under various external parameters.

The subject of the research was the basic production cycle developed and investigated. The cycle covered the fabrication of a nanoceramic sample out of a nanopowder up to the consolidation process of the nanomaterial, the mechanisms and models of structural and phase transformations, considering the diffusion processes and crystallization phenomena in nanoceramics.

The fundamental problem to be solved was to obtain the energy-saving consolidation nanoceramics powder compositions in a tightly packed product with improved or special functional properties. This problem was solved by means of the intensification of nanopowders consolidation process and accordingly the reduction of high-temperature stage time. The investigations led to the conclusion that the most effective method was to activate the sintering with the electromagnetic field applied for heating nanopowder particles. It appeared that the method of the electrical consolidation by the direct passing of the current was very effective in case of conductive nanopowders sintering. In the case of nonconducting nanopowders, rather a microwave sintering is applied [11], where the heating process of dielectric losses under the influence of high-frequency electric field takes place. In both cases, usually high heating rates are used.

The investigation proved, however, that the electrical consolidation can be used with equally good result for both conductive and non-conductive powders (through the use of electrically conductive molds) and microwave sintering has been successfully used for the sintering of ceramics and metals. This confirms the urgent need for fundamental research on the activated electric consolidation of nanostructured bodies primarily through theoretical analysis and simulation experiments, quite rarely used today in the field of materials science.

The Figures $7 \mathrm{a}$ and $7 \mathrm{~b}$ present the examples of the electrically sintered microstructures of zinc sulfide and titanium dioxide ceramics, respectively.

a)

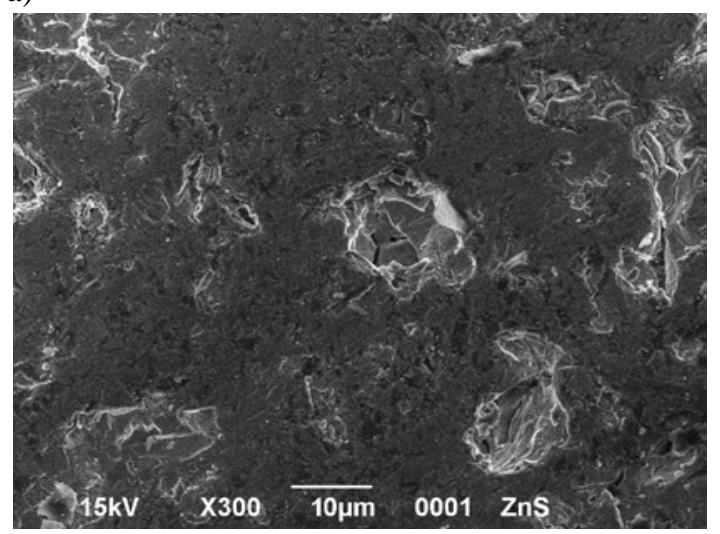

b)

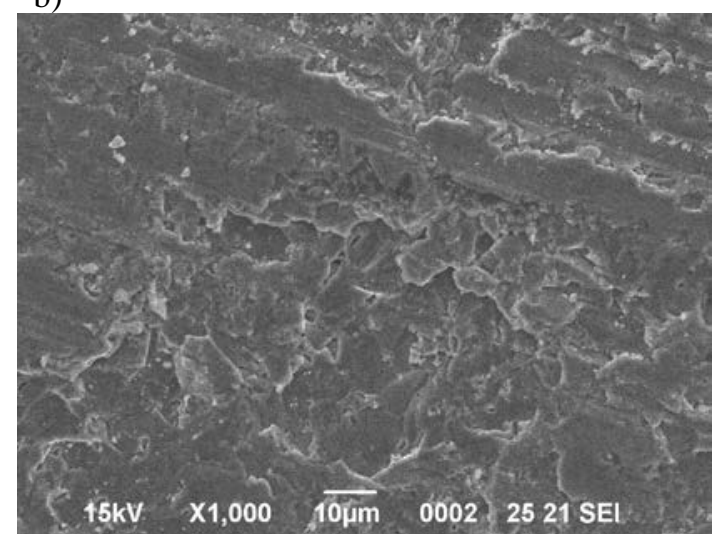

Figure 7. Photomicrographs: a) of zinc sulfide ceramic structure after electrical consolidation at $1360^{\circ} \mathrm{C}$; b) of titanium dioxide ceramic structure after electrical consolidation at $1200^{\circ} \mathrm{C}$.

The parameters of the hot pressing process conducted under the electric current, as well as the sintered material analysis results, revealed highly desirable characteristics. For example, in the proposed novel method, the theoretical density values for the $\mathrm{Al}_{2} \mathrm{O}_{3}$ ceramics were obtained in $t=2$ min at the temperature $T=1130{ }^{\circ} \mathrm{C}$, while the recent studies reported that the ceramic with $97.6 \%$ of theoretical density and $1.1 \mu \mathrm{m}$ of average grain size was obtained at $1550{ }^{\circ} \mathrm{C}$ [12]. In another experiment, the 2 minutes of sintering of the tungsten carbide at the temperature below $1800^{\circ} \mathrm{C}$ produced the samples of the flexural strength ca. $\sigma_{f}=720 \mathrm{MPa}$. It is very advantageous compared to the results obtained for 
the high temperature sintering at $T=2500{ }^{\circ} \mathrm{C}$ during $t=10 \mathrm{~min}$, where the flexural strength was at best MPa [13].

\section{Conclusions and Future Researches}

The research on the nanopowders synthesis provided high quality results. The X-ray diagrams of the $\mathrm{ZnS}$ powders obtained with microwave activation at any $\mathrm{pH}$, are identical to the diagram of the ZnS powder obtained without it at $\mathrm{pH}$ 12. On the other hand, the IR spectra are quite similar to the ones obtained for the powders produced without microwave activation. The analysis indicated the absence of the zinc oxide in the microwave activated powder. The yield of zinc sulfite produced with the microwave activation is much higher, and reaches ca. $90 \%$ irrespectively of $\mathrm{pH}$ during the synthesis process. Further researches are directed to the detailed investigations of the $\mathrm{ZrO}_{2}$ nanopowders.

In case of the high-speed sintering under the high temperature pressing, with the directly applied alternating current, it is possible to achieve the density of the sintered material close to the theoretical value even at the temperature $1100^{\circ} \mathrm{C}$ and in $20 \%$ of the typical processing time. The electrical consolidation can be used with equally good result for both conductive and non-conductive powders.

\section{References}

1. M. Cain and R. Morell, "Nanostructured ceramics: a review of their potential," Applied Organometallic Chemistry, vol. 15, pp. 321-330, 2001.

2. H. Hahn, "Nanostructured ceramics: microstructure and properties," in Nanophase Materials. Kluwer Academic Publishers, 1994, pp. 263-273.

3. N. Camargo, O.J. Bellini, E. Gemelli and M. Tomiyama, "Synthesis and characterization of nanostructured ceramics powders for biomedical applications," Matéria (Rio J.), vol.12, no.4, pp. 574-582, 2007.

4. A.S.H. Makhlouf and D. Scharnweber, Handbook of Nanoceramic and Nanocomposite Coatings and Materials. Elsevier-BH, 2015.

5. A. Guglya, A. Kalchenko, E. Solopikhina, V. Vlasov and E. Lyubchenko, "Nanocrystalline Porous Thin Film VNx Hydrogen Absorbents: Method of Production, Structure and Properties," Journal of Advances in Nanomaterials, vol. 1, no. 1, pp. 1-10, 2016.

6. M. Van de Voorde, M. Werner and H.J. Fecht (eds.), The Nano-Micro Interface. Wiley-VCH, 2015.

7. M. Nanko, and K.Q. Dang, "Two-step pulsed electric current sintering of transparent $\mathrm{Al}_{2} \mathrm{O}_{3}$ ceramics," Advances in Applied Ceramics, vol. 13, no. 2, pp. 80-84, 2014.

8. L. Theodore and R.G. Kunz, Nanotechnology: Environmental Implications and Solutions. Wiley-Interscience, 2005.

9. T.Y. Tseng and H.S. Nalwa (eds.), Handbook of Nanoceramics and Their Based Nanodevices. American Scientific Publishers, 2009.

10.A. I. Gusev, Nanomaterials, Nanostructures, and Nanotechnologies (in Russian). Fizmatlit, 2007.

11. P. Sharma, J.D. Majumdar, "Studies on nano-crystalline CoNiCrAlY consolidated by conventional and microwave sintering," Advanced Powder Technology, vol. 27, no. 1, pp. 72-84, 2016.

12.Zh. Suna, B. Lia, P. Hua, F. Dinga and F. Yuan, "Alumina ceramics with uniform grains prepared from $\mathrm{Al}_{2} \mathrm{O}_{3}$ nanospheres," Journal of Alloys and Compounds, vol. 688, Part A, pp. 933-938, 2016.

13.K. Niihara, "Mechanical properties of chemically vapor deposites nanoxide ceramics," American Ceramic Society Bulletin, vol. 19, no. 9, pp. 1160-1164, 1984. 